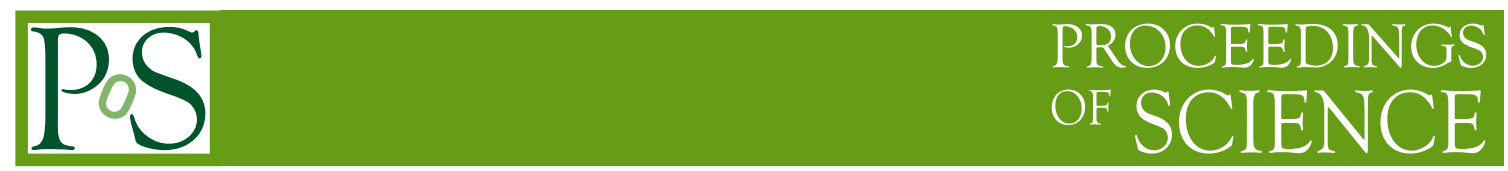

\title{
Sidereal anisotropy of Galactic cosmic ray observed by the Tibet Air Shower experiment and the IceCube experiment
}

M. Amenomori ${ }^{1}$, X. J. Bi ${ }^{2}$, D. Chen ${ }^{3}$, T. L. Chen ${ }^{4}$, W. Y. Chen ${ }^{2}$, S. W. Cui ${ }^{5}$,

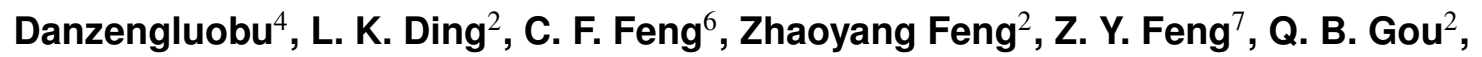
Y. Q. Guo ${ }^{2}$, H. H. He ${ }^{2}$, Z. T. He ${ }^{5}$, K. Hibino ${ }^{8}$, N. Hotta ${ }^{9}$, Haibing $\mathrm{Hu}^{4}$, H. B. $\mathrm{Hu}^{2}$, J. Huang ${ }^{2}$, H. Y. Jia ${ }^{7}$, L. Jiang ${ }^{2}$, F. Kajino ${ }^{10}$, K. Kasahara ${ }^{11}$, Y. Katayose ${ }^{12}$, C. Kato ${ }^{13}$, K. Kawata ${ }^{14}$, M. Kozai ${ }^{13}$, Labaciren ${ }^{4}$, G. M. Le ${ }^{15}$, A. F. Li ${ }^{16,6,2}$, H. J. Li ${ }^{4}$, W. J. Li ${ }^{2,7}$, C. Liu $^{2}$, J. S. Liu ${ }^{2}$, M. Y. Liu ${ }^{4}$, H. Lu ${ }^{2}$, X. R. Meng ${ }^{4}$, T. Miyazaki ${ }^{13}$, K. Mizutani ${ }^{11,17}$, K. Munakata ${ }^{13}$, T. Nakajima ${ }^{13}$, Y. Nakamura ${ }^{13}$, H. Nanjo ${ }^{1}$, M. Nishizawa ${ }^{18}$, T. Niwa ${ }^{13}$, M. Ohnishi ${ }^{14}$, I. Ohta ${ }^{19}$, S. Ozawa ${ }^{11}$, X. L. Qian ${ }^{6,2}$, X. B. Qu ${ }^{20}$, T. Saito ${ }^{21}$, T. Y. Saito ${ }^{22}$, M. Sakata ${ }^{10}$, T. K. Sako ${ }^{14}$, J. Shao ${ }^{2,6}$, M. Shibata ${ }^{12}$, A. Shiomi ${ }^{23}$, T. Shirai ${ }^{8}$, H. Sugimoto ${ }^{24}$, M. Takita ${ }^{14}$, Y. H. Tan ${ }^{2}$, N. Tateyama ${ }^{8}$, S. Torii ${ }^{11}$, H. Tsuchiya ${ }^{25}$, S. Udo ${ }^{8}$, H. Wang ${ }^{2}$, H. R. $\mathbf{W u}^{2}$, L. Xue ${ }^{6}$, Y. Yamamoto ${ }^{10}$, K. Yamauchi ${ }^{12}$, Z. Yang ${ }^{2}$, S. Yasue ${ }^{26}$, A. F. Yuan ${ }^{4}$, T. Yuda ${ }^{14}$, L. M. Zhai ${ }^{2}$, H. M. Zhang ${ }^{2}$, J. L. Zhang ${ }^{2}$, X. Y. Zhang ${ }^{6}$, Y. Zhang ${ }^{2}$, Yi Zhang ${ }^{2}$, Ying Zhang ${ }^{2}$ Zhaxisangzhu $^{4}$, X. X. Zhou ${ }^{7}$ 


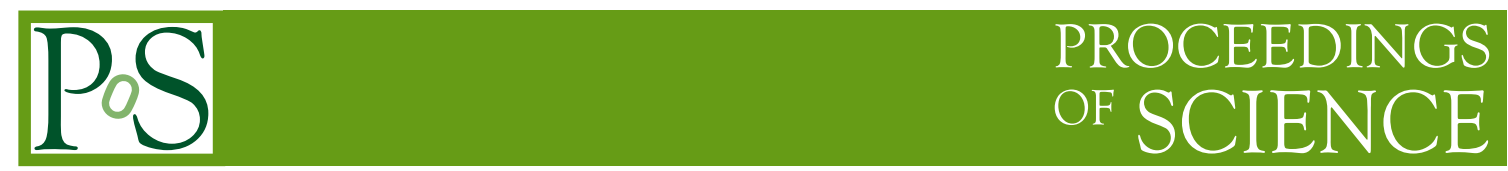

(The Tibet AS $\gamma$ Collaboration)

${ }^{1}$ Department of Physics, Hirosaki University, Hirosaki 036-8561, Japan

${ }^{2}$ Key Laboratory of Particle Astrophysics, Institute of High Energy Physics, Chinese Academy of Sciences, Beijing 100049, China

${ }^{3}$ National Astronomical Observatories, Chinese Academy of Sciences, Beijing 100012, China

${ }^{4}$ Department of Mathematics and Physics, Tibet University, Lhasa 850000, China

${ }^{5}$ Department of Physics, Hebei Normal University, Shijiazhuang 050016, China

${ }^{6}$ Department of Physics, Shandong University, Jinan 250100, China

${ }^{7}$ Institute of Modern Physics, SouthWest Jiaotong University, Chengdu 610031, China

${ }^{8}$ Faculty of Engineering, Kanagawa University, Yokohama 221-8686, Japan

${ }^{9}$ Faculty of Education, Utsunomiya University, Utsunomiya 321-8505, Japan

${ }^{10}$ Department of Physics, Konan University, Kobe 658-8501, Japan

${ }^{11}$ Research Institute for Science and Engineering, Waseda University, Tokyo 169-8555, Japan

${ }^{12}$ Faculty of Engineering, Yokohama National University, Yokohama 240-8501, Japan

${ }^{13}$ Department of Physics, Shinshu University, Matsumoto 390-8621, Japan

${ }^{14}$ Institute for Cosmic Ray Research, University of Tokyo, Kashiwa 277-8582, Japan

${ }^{15}$ National Center for Space Weather, China Meteorological Administration, Beijing 100081, China

${ }^{16}$ School of Information Science and Engineering, Shandong Agriculture University, Taian 271018, China

${ }^{17}$ Saitama University, Saitama 338-8570, Japan

${ }^{18}$ National Institute of Informatics, Tokyo 101-8430, Japan

${ }^{19}$ Sakushin Gakuin University, Utsunomiya 321-3295, Japan

${ }^{20}$ College of Science, China University of Petroleum, Qingdao 266555, China

${ }^{21}$ Tokyo Metropolitan College of Industrial Technology, Tokyo 116-8523, Japan

${ }^{22}$ Max-Planck-Institut für Physik, München D-80805, Deutschland

${ }^{23}$ College of Industrial Technology, Nihon University, Narashino 275-8576, Japan

${ }^{24}$ Shonan Institute of Technology, Fujisawa 251-8511, Japan

${ }^{25}$ Japan Atomic Energy Agency, Tokai-mura 319-1195, Japan

${ }^{26}$ School of General Education, Shinshu University, Matsumoto 390-8621, Japan

We analyze for the first time the large-scale sidereal anisotropy of $\sim 10 \mathrm{TeV}$ cosmic ray intensity observed in two hemispheres, by best fitting a model to the anisotropies observed with the Tibet AS array in the northern hemisphere and the IceCube in the southern hemisphere. We find that the first and second harmonics of the right ascension distribution observed in two hemispheres are consistent with each other and well reproduced by a model of the superposed uni-directional and bi-directional cosmic ray flows. It is also shown that the best-fit amplitude and orientation of each flow are consistent with the best-fit parameters reported so far by the Tibet AS experiment.

The 34th International Cosmic Ray Conference

30 July- 6 August, 2015

The Hague, The Netherlands 


\section{1. introduction}

Recent underground muon and air-shower (AS) experiments including the Tibet AS experiment have consistently reported the large-scale (or global) features of the sidereal anisotropy of cosmic ray intensity in multi- $\mathrm{TeV}$ to $\sim 10 \mathrm{TeV}$ region, suggesting that there are two distinct broad structures in the anisotropy with an amplitude of $\sim 10^{-3}(0.1 \%)$; one is a deficit in the cosmic-ray flux centered around 150 to 240 degree in Right Ascension (RA); the other is an excess in the cosmic-ray flux around 40 to 90 in RA (Amenomori et al., 2006 [四; Guillian et al., 2007 []]; Abdo et al., 2009 [3] $)$. The observed anisotropy of galactic cosmic rays at $\mathrm{TeV}$ energies is considered to reflect magnetic field structures of the heliosphere and/or the local interstellar space, since the Larmor radius of cosmic rays (predominantly protons) in this energy regions is comparable to or smaller than the scale size of these regions.

Amenomori et al. (2007 [四], 2010 []]) expressed the anisotropy as a combination of the unidirectional and the bi-directional flows of galactic cosmic rays. The bi-directional flow is produced by cosmic rays drifting parallel to the local interstellar magnetic field line into the heliosphere from outside the Local Interstellar Cloud (LIC) surrounding the heliosphere. On the other hand, the uni-directional flows, which is perpendicular to the field line, can be produced by a diamagnetic drift arising from a spatial density gradient of galactic cosmic rays in the LIC. Recent works of Zhang et al. (2014) [焑] and Schwadron et al. (2014) [四] also discussed the local origin model of the anisotropies, while Qu et al. (2012) [8] proposed a global galactic" CR Stream " model to understand the observation of the major anisotropic components in the solar vicinity. Many related studies are ongoing, but a generally accepted model or theory capable of interpreting all the observations does not exist yet. More data and analyses are necessary to provide a solid ground for a firm theory.

We note in the present paper that the most of experiments referred above report the anisotropy observed in the northern sky and the observation in the southern sky is still seldom. Recently, Abbasi et al. (2010 [Q] , 2012 [ए]]) reported the anisotropy of high energy muon intensity observed with the IceCube experiment in operation at the south-pole. They supplied in a table of their paper the first and second harmonics of the RA distribution of $20 \mathrm{TeV}$ cosmic rays in each declination (DEC) band. In this paper, we combine the harmonics reported by the IceCube with those observed with the Tibet AS array and perform a best-fit analysis of a model anisotropy to the combined harmonics data in two hemispheres.

\section{Data analysis and result}

\subsection{Harmonic analysis of RA distribution in each DEC band}

Abbasi et al. (2012) [ए]] (referred as paper 1 hereafter) presented the first and second harmonics of the RA distribution of $20 \mathrm{TeV}$ cosmic ray intensity in each declination (DEC) band (see Table 1 in their paper). Open squares in Figure $\square$ show the energy distribution of primary cosmic rays observed by the IceCube, which are read by us from Figure 3 of paper 1. The median primary energy in this distribution is $20 \mathrm{TeV}$. 
To analyze the anisotropy observed with the Tibet AS array in the same energy region, we collect air shower events which satisfy the following four conditions, (i) any four-fold coincidence occurs in counters with each recording more than 1.25 particles in charge, (ii) the air shower core position is located in the array, (iii) the zenith angle of arrival direction is $\leq 60^{\circ}$, (iv) $\sum \rho_{F T}$ which denotes the sum of number of particles per $\mathrm{m}^{2}$ for each FT detector must satisfy $63 \leq \sum \rho_{F T} \leq 160$. Under these conditions, total $9.0 \times 10^{9}$ events are collected with the median primary energy of $14 \mathrm{TeV}$ during a period from November 1999 through May 2010. The energy distribution of these events is shown by

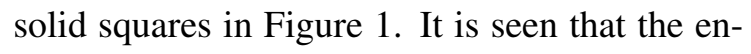
ergy distributions by the Tibet AS array and the IceCube are well overlapping with each other.

We analyze the first and second harmonics

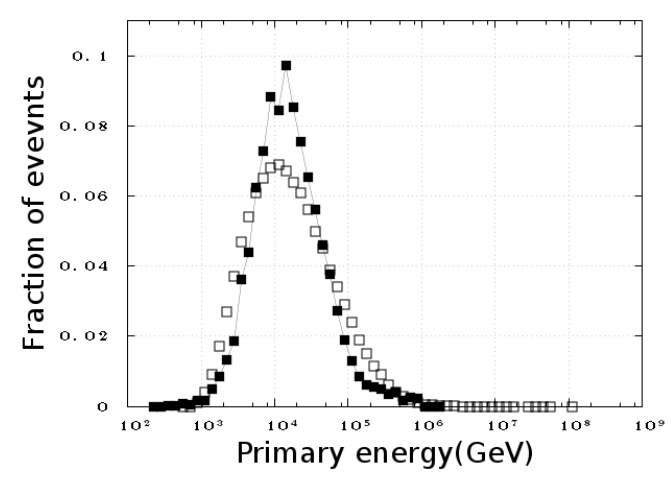

Figure 1: Energy distribution of primary cosmic rays observed by the Tibet AS array and the IceCube. Normalized event numbers observed by the Tibet AS array (solid square) and the IceCube (open square) are plotted each as a function of primary energy on the horizontal axis in a logarithmic scale. The data of the IceCube experiment are read from Figure 3 of paper 1 [四].

of the RA distribution of cosmic ray intensity observed in two hemispheres with the Tibet AS array and IceCube. After the normalization in each DEC band, the cosmic ray intensity $I(\alpha, \delta)$ at the RA $\alpha$ and DEC $\delta$ is expressed in terms of the first and second harmonics, as

$$
\begin{aligned}
I(\alpha, \boldsymbol{\delta}) & =1+\sum_{i=1}^{2}\left\{a_{i}(\boldsymbol{\delta}) \cos (i \alpha)+b_{i}(\boldsymbol{\delta}) \sin (i \alpha)\right\} \\
& =1+\sum_{i=1}^{2} A_{i}(\boldsymbol{\delta}) \cos \left(i\left(\alpha-\alpha_{i}(\boldsymbol{\delta})\right)\right.
\end{aligned}
$$

The harmonic coefficients $a_{i}(\boldsymbol{\delta})$ and $b_{i}(\boldsymbol{\delta})$ are obtained from the observed intensity, as

$$
\begin{aligned}
& a_{i}(\boldsymbol{\delta})=\frac{2}{N} \sum_{k=1}^{N} I_{o b s}(\omega k, \delta) \cos (i \omega k) \\
& b_{i}(\delta)=\frac{2}{N} \sum_{k=1}^{N} I_{o b s}(\omega k, \delta) \sin (i \omega k)
\end{aligned}
$$

where $I_{o b s}(\omega k, \delta)$ is the observed normalized intensity, $N$ is the number of RA bins in each DEC band, and $\omega=2 \pi / N$. We obtain $a_{i}(\delta)$ and $b_{i}(\delta)$ by the IceCube experiment from the amplitude $\left(A_{i}(\delta)\right.$ )and phase $\left(\alpha_{i}(\delta)\right)$ of the harmonics given in Table 1 of paper 1 , as

$$
\begin{aligned}
& a_{i}(\boldsymbol{\delta})=A_{i}(\boldsymbol{\delta}) \cos \left(i \alpha_{i}(\boldsymbol{\delta})\right) \\
& b_{i}(\boldsymbol{\delta})=A_{i}(\boldsymbol{\delta}) \sin \left(i \alpha_{i}(\boldsymbol{\delta})\right) .
\end{aligned}
$$




\subsection{Statistical and systematic errors of harmonic coefficients}

The statistical errors of $a_{i}(\boldsymbol{\delta})$ and $b_{i}(\boldsymbol{\delta})(i=1,2)$ by Tibet AS experiment are propagated from statistical error of $I_{o b s}(\alpha, \delta)$ in Eqs. 2.2 and 2.3 , while the statistical errors of $a_{i}(\delta)$ and $b_{i}(\delta)$ by the IceCube experiment are propagated from the statistical errors of $A_{i}$ and $\alpha_{i}(\delta)$ given in Table 1 of paper 1. For systematical error of $a_{i}(\delta)$ and $b_{i}(\delta)$ by Tibet AS experiment, we use harmonic coefficients $a_{i}^{A S}(\delta)$ and $b_{i}^{A S}(\delta)$ observed in the anti-sidereal time in each DEC band, as

$$
\begin{aligned}
& \sigma a_{i}^{\text {sys }}(\boldsymbol{\delta})=a_{i}^{A S}(\boldsymbol{\delta})+\sigma a_{i}(\boldsymbol{\delta}) \\
& \sigma b_{i}^{\text {sys }}(\boldsymbol{\delta})=b_{i}^{A S}(\boldsymbol{\delta})+\sigma b_{i}(\boldsymbol{\delta})
\end{aligned}
$$

where $\sigma a_{i}(\boldsymbol{\delta})$ and $\sigma b_{i}(\boldsymbol{\delta})$ are statistical error of $a_{i}^{A S}(\boldsymbol{\delta})$ and $b_{i}^{A S}(\boldsymbol{\delta})$.

For the systematic errors of $a_{i}(\delta)$ and $b_{i}(\delta)$ by IceCube experiment, we use the amplitude $A_{i}^{A S_{-} 1 D}$ of the one-dimensional (1D) RA distribution observed in the anti-sidereal time and given in Table 5 of paper 1 in addition to the statistical error $\sigma A_{i}(\delta)$ of the harmonic amplitude in each DEC band, as

$$
\sigma a_{i}^{\text {sys }}(\boldsymbol{\delta})=\sigma b_{i}^{\text {sys }}(\boldsymbol{\delta})=A_{i}^{A S_{-} 1 D}+\sigma A_{i}(\boldsymbol{\delta}) .
$$

\subsection{Best-fit model}

We best-fit the model anisotropy consisting of uni-directional flow (UDF) and bi-directional flow (BDF) to the harmonic coefficients observed by the Tibet AS array and IceCube in each DEC band and derive the best-fit parameters of the model. In this model, the normalized intensity $I^{\exp }\left(\alpha, \delta ; A_{1}, \alpha_{1}, A_{2}, \alpha_{2}, \delta_{2}\right)$ expected at $\alpha$ and $\delta$ is given, as

$$
\begin{aligned}
I^{\exp }(\alpha, \delta & \left.; A_{1}, \alpha_{1}, A_{2}, \alpha_{2}, \delta_{2}\right) \\
=1 & +A_{1} P_{1}^{0}\left(\cos D_{1}\right)+A_{2} P_{2}^{0}\left(\cos D_{2}\right) \\
=1 & +\left(x_{1}^{1} \cos \alpha+y_{1}^{1} \sin \alpha\right) \times P_{1}^{1}\left(\cos \left(\frac{\pi}{2}-\delta\right)\right) \\
& +\left(x_{2}^{1} \cos \alpha+y_{2}^{1} \sin \alpha\right) \times P_{2}^{1}\left(\cos \left(\frac{\pi}{2}-\delta\right)\right) \\
& +\left(x_{2}^{2} \cos 2 \alpha+y_{2}^{2} \sin 2 \alpha\right) \times P_{2}^{2}\left(\cos \left(\frac{\pi}{2}-\delta\right)\right)
\end{aligned}
$$

where $A_{1}$ and $\alpha_{1}$ are parameters denoting the amplitude and the RA of the reference axis of the UDF, respectively, $A_{2}, \alpha_{2}$ and $\delta_{2}$ are parameters denoting the amplitudes, the RA and DEC of the reference axis of the BDF, respectively, and $D_{1}$ and $D_{2}$ are angles of the viewing direction $(\alpha, \delta)$ from the reference axes of the UDF and BDF, respectively. Since the DEC of the UDF reference axis $\delta_{1}$ cannot be derived from the intensity normalized in each DEC band, we set $\delta_{1}=0$. In Eq. ([.] $), P_{1}^{0}, P_{2}^{0}, P_{1}^{1}, P_{2}^{1}$ and $P_{2}^{2}$ are the Schmidt semi-normalized associated Legendre functions, defined as

$$
P_{n}^{m}(\cos \theta)= \begin{cases}P_{n, m}(\cos \theta) & \text { for } m=0 \\ \sqrt{\frac{2(n-m) !}{(n+m) !}} P_{n, m}(\cos \theta) & \text { for } m \neq 0\end{cases}
$$


and

$$
P_{n, m}=\sin ^{m} \theta \frac{d^{m} P_{n}(\cos \theta)}{d(\cos \theta)^{m}} .
$$

Angles $D_{1}$ and $D_{2}$ in Eq. (2.7) are expressed in terms of $\alpha_{1}, \alpha_{2}, \delta_{2}, \alpha$ and $\delta$, as

$$
\begin{aligned}
\cos D_{1} & =\sin \left(\frac{\pi}{2}-\delta\right) \sin \left(\frac{\pi}{2}\right) \cos \left(\alpha-\alpha_{1}\right) \\
\cos D_{2} & =\cos \left(\frac{\pi}{2}-\delta\right) \cos \left(\frac{\pi}{2}-\delta_{2}\right) \\
& +\sin \left(\frac{\pi}{2}-\delta\right) \sin \left(\frac{\pi}{2}-\delta_{2}\right) \cos \left(\alpha-\alpha_{2}\right) .
\end{aligned}
$$

Six parameters, $x_{1}^{1}, y_{1}^{1}, x_{2}^{1}, y_{2}^{1}, x_{2}^{2}$ and $y_{2}^{2}$ in Eq. (‥ (]) are expressed in terms of $A_{1}, \alpha_{1}, A_{2}, \alpha_{2}, \delta_{2}$, as

$$
\begin{aligned}
x_{1}^{1} & =A_{1} P_{1}^{1}\left(\cos \left(\frac{\pi}{2}-\delta_{1}\right)\right) \cos \alpha_{1} \\
y_{1}^{1} & =A_{1} P_{1}^{1}\left(\cos \left(\frac{\pi}{2}-\delta_{1}\right)\right) \sin \alpha_{1} \\
x_{2}^{1} & =A_{2} P_{2}^{1}\left(\cos \left(\frac{\pi}{2}-\delta_{2}\right)\right) \cos \alpha_{2} \\
y_{2}^{1} & =A_{2} P_{2}^{1}\left(\cos \left(\frac{\pi}{2}-\delta_{2}\right)\right) \sin \alpha_{2} \\
x_{2}^{2} & =A_{2} P_{2}^{2}\left(\cos \left(\frac{\pi}{2}-\delta_{2}\right)\right) \cos 2 \alpha_{2} \\
y_{2}^{2} & =A_{2} P_{2}^{2}\left(\cos \left(\frac{\pi}{2}-\delta_{2}\right)\right) \sin 2 \alpha_{2} .
\end{aligned}
$$

The first and second harmonic coefficients, $a_{1}^{\exp }(\boldsymbol{\delta}), b_{1}^{\exp }(\boldsymbol{\delta}), a_{2}^{\exp }(\boldsymbol{\delta})$ and $b_{2}^{\exp }(\boldsymbol{\delta})$, of $I^{\exp }\left(\alpha, \delta ; A_{1}, \alpha_{1}, A_{2}, \alpha_{2}, \delta_{2}\right)$ in each DEC band are given by six parameters in Eq. (․ㅣ) , as

$$
\begin{aligned}
& a_{1}^{e x p}(\delta)=x_{1}^{1} P_{1}^{1}\left(\cos \left(\frac{\pi}{2}-\delta\right)\right)+x_{2}^{1} P_{2}^{1}\left(\cos \left(\frac{\pi}{2}-\delta\right)\right) \\
& b_{1}^{e x p}(\delta)=y_{1}^{1} P_{1}^{1}\left(\cos \left(\frac{\pi}{2}-\delta\right)\right)+y_{2}^{1} P_{2}^{1}\left(\cos \left(\frac{\pi}{2}-\delta\right)\right) \\
& a_{2}^{e x p}(\delta)=x_{2}^{2} P_{2}^{2}\left(\cos \left(\frac{\pi}{2}-\delta\right)\right) \\
& b_{2}^{e x p}(\delta)=y_{2}^{2} P_{2}^{2}\left(\cos \left(\frac{\pi}{2}-\delta\right)\right) .
\end{aligned}
$$

As seen in Eq. (2.12), this model expresses the first harmonic coefficient $a_{1}^{\exp }\left(b_{1}^{\exp }\right)$ in terms of a combination of the north-south symmetric and anti-symmetric DEC distributions, $P_{1}^{1}$ and $P_{2}^{1}$, with amplitudes $x_{1}^{1}$ and $x_{2}^{1}\left(y_{1}^{1}\right.$ and $\left.y_{2}^{1}\right)$, respectively, while it expresses the second harmonic coefficient $a_{2}^{\text {exp }}\left(b_{2}^{\text {exp }}\right)$ by only the north-south symmetric DEC distribution, $P_{2}^{2}$, with an amplitude $x_{2}^{2}\left(y_{2}^{2}\right)$. By comparing $a_{i}^{e x p}(\boldsymbol{\delta})$ and $b_{i}^{e x p}(\boldsymbol{\delta})$ with the observed $a_{i}(\boldsymbol{\delta})$ and $b_{i}(\boldsymbol{\delta})$ in Eqs. (2.2)-(2.4), we obtain six best-fit parameters $\left(x_{1}^{1}, y_{1}^{1}, x_{1}^{2}, y_{1}^{2}, x_{2}^{2}, y_{2}^{2}\right)$ minimizing the residual $\chi^{2}$ defined as

$$
\begin{aligned}
\chi^{2}=\sum_{\delta}\left\{\frac{\left(a_{1}(\boldsymbol{\delta})-a_{1}^{e x p}(\boldsymbol{\delta})\right)^{2}}{\left(\sigma a_{1}(\boldsymbol{\delta})+\sigma a_{1}^{s y s}(\boldsymbol{\delta})\right)^{2}}+\frac{\left(b_{1}(\boldsymbol{\delta})-b_{1}^{e x p}(\boldsymbol{\delta})\right)^{2}}{\left(\sigma b_{1}(\boldsymbol{\delta})+\sigma b_{1}^{s y s}(\boldsymbol{\delta})\right)^{2}}\right. \\
\left.+\frac{\left(a_{2}(\boldsymbol{\delta})-a_{2}^{e x p}(\boldsymbol{\delta})\right)^{2}}{\left(\sigma a_{2}(\boldsymbol{\delta})+\sigma a_{2}^{\text {sys }}(\boldsymbol{\delta})\right)^{2}}+\frac{\left(b_{2}(\boldsymbol{\delta})-b_{2}^{\text {exp }}(\boldsymbol{\delta})\right)^{2}}{\left(\sigma b_{2}(\boldsymbol{\delta})+\sigma b_{2}^{\text {sys }}(\boldsymbol{\delta})\right)^{2}}\right\}
\end{aligned}
$$



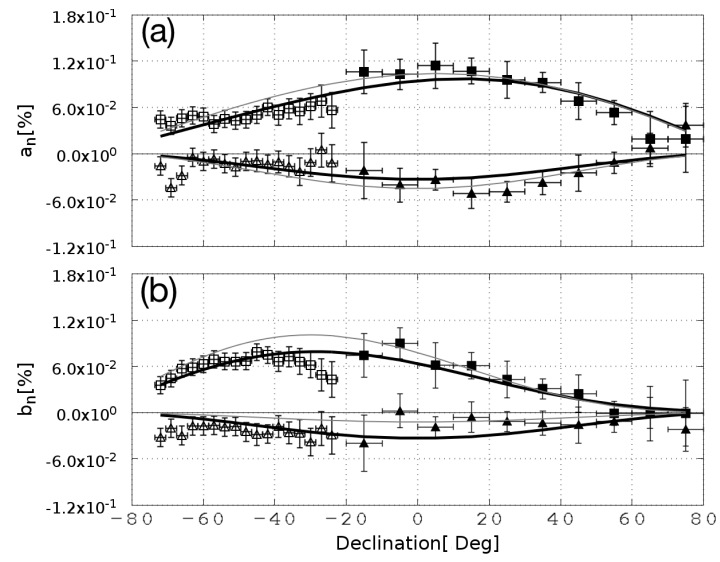
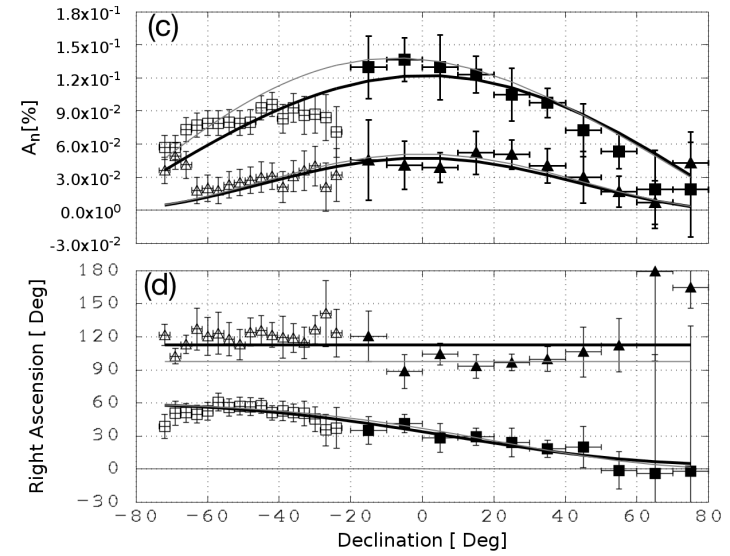

Figure 2: First and second harmonics of the RA distribution in each DEC band observed by the IceCube and the Tibet AS array. The first harmonics observed by the Tibet AS array (IceCube) are plotted by solid (open) squares, while the second harmonics are plotted by solid (open) triangles. The error of each data point includes the statistical and systematic errors evaluated in section 2.2 (see text). Panels (a) and (b) display the observed harmonic coefficients, $a_{i}(\boldsymbol{\delta})$ and $b_{i}(\boldsymbol{\delta})$, while panels (c), (d) show the observed amplitude and phase, $A_{i}(\delta)$ and $\alpha_{i}(\delta)$, each as a function of the DEC on the horizontal axis. The solid (light) curve in each panel shows the DEC dependence expected from the model anisotropy best-fitting to both of the IceCube and Tibet AS data (the Tibet AS data alone).

\subsection{Result}

Solid (open) squares in Figure 2 display the first harmonic coefficients of the RA distribution observed by Tibet AS array (IceCube), while solid (open) triangles display the second harmonic coefficients of the RA distribution observed by Tibet AS array (IceCube). The DEC dependences of $a_{i}(\boldsymbol{\delta})$ and $b_{i}(\boldsymbol{\delta})$ expected from the model best-fitting to both data by the Tibet AS and IceCube experiments are also shown by solid curves, while the light curves show the DEC dependences expected from the best-fitting only to the Tibet AS experiment data. Due to a notable north-south asymmetry seen in panels (a) and (b), the observed phase of the first harmonic in panel (d) tends to shift to larger RA with the DEC moving toward south over the equator. This asymmetry is also seen in the best-fit curve derived from the Tibet AS array data alone. A similar north-south asymmetry has been already reported by Munakata et al. (2010) [12] from the two hemisphere observations of sub- $\mathrm{TeV}$ cosmic rays by a pair of multi-directional underground muon detectors in Japan and Australia, while the best-fit amplitudes reported by Munakata et al. (2010) are only one fifth of the amplitudes in Table 1 due to the attenuation by the solar modulation.

Table 1: Model parameters obtained from the best-fit analyses. The first line presents the parameters obtained from best-fitting to the Tibet AS array data alone, while the second line presents the parameters from best-fitting to both of the IceCube and Tibet AS data.

\begin{tabular}{ccccccc}
\hline data used & $A_{1}[\%]$ & $\alpha_{1}[\mathrm{deg}]$ & $A_{2}[\%]$ & $\alpha_{2}[\mathrm{deg}]$ & $\delta_{2}[\mathrm{deg}]$ & $\chi^{2} /$ d.o.f \\
\hline Tibet only & 0.13 & 39.96 & 0.07 & 97.5 & -22.5 & $10.5 /(40-5)$ \\
Tibet \& IceCube & 0.11 & 33.95 & 0.07 & 112.5 & -17.5 & $82.0 /(108-5)$ \\
\hline
\end{tabular}




\section{3. summary}

We analyzed the first and second harmonics of the RA distributions observed in two hemispheres by the Tibet AS array at $14 \mathrm{TeV}$ and by IceCube at $20 \mathrm{TeV}$. We find the north-south asymmetry in the DEC dependences of the first harmonic coefficients, while the DEC dependences of the second harmonic coefficients are consistent with the north-south symmetry. From a best-fit analyses to the observed harmonic coefficients, we find that the observed DEC dependences can be reproduced from the model anisotropy consisting of the UDF and BDF. It is also found that the best-fit parameters of the UDF and BDF are consistent with the results reported by the Tibet AS experiment [ए]] and two hemisphere observations with underground muon detectors at sub-TeV region [ए2].

\section{Acknowlegments}

The collaborative experiment of the Tibet Air Shower Arrays has been performed under the auspices of Ministry of Science and Technology of China and Ministry of Foreign Priority Affairs of Japan. This work was supported in part by a Grant-in-Aid for Science Research from the Ministry of Education, Culture, Sports, Science and Technology, by Geants-in-Aid for Science Research from the Japan Society for Promotion of Science in Japan, and by the Grants from the National Natural Science Foundation of China and Chinese Academy of Sciences.

\section{References}

[1] Amenomori, M., et al., 2006, Science 314, 439.

[2] Guillian, G., et al., 2007, Phys. Rev. D. 75, 062003.

[3] Abdo, A. A., et al, 2009, ApJ 698, 2121.

[4] Amenomori, M., et al., 2007, AIP Conf. Proc. 932, 283.

[5] Amenomori, M., et al., 2010, AIP Conf. Proc. 1302, 285.

[6] Zhang, M., et al., 2014, ApJ 790, 5.

[7] Schwadron, N. A., et al., 2014, Science 343, 988.

[8] Qu, X. B., et al., 2012, ApJL 750, L1.

[9] Abassi, R., et al., 2010, ApJL 718, L194.

[10] Abbasi, R., et al., 2012, ApJ 746, 33.

[11] Amenomori, M., et al., 2010, Astrophys. Space Sci. Trans. 6, 49.

[12] Munakata, K., et al., 2010, Advaces in Geosciense 21, 211. 